\title{
A NOTE ON THE CONTINUITY OF MULTILINEAR MAPPINGS IN TOPOLOGICAL MODULES
}

\author{
NILSON C. BERNARDES JR. \\ Instituto de Matemática \\ Universidade Federal Fluminense \\ Rua São Paulo s/n \\ 24210 Rio de Janeiro \\ BRASIL
}

(Received November 11, 1993 and in revised form December 15, 1993)

\begin{abstract}
In the present note, we obtain a criterion for the equicontinuity of families of multilinear mappings between topological modules. We also give an example which shows that the hypothesis imposed on the neighborhoods of zero is essential for the validity of our theorem.
\end{abstract}

KEY WORDS AND PHRASES. Equicontinuity, multilinear mappings, topological modules. 1991 AMS SUBJECT CLASSIFICATION CODE(S). $46 \mathrm{H} 25$.

\section{INTRODUCTION.}

Our goal is to establish the below generalization of a classical result of Functional Analysis ([1], chap. I, §1, proposition 6).

\section{MAIN RESULTS.}

THEOREM. Let $\mathrm{A}$ be a commutative topological ring, $E_{1}, \cdots, E_{n}, F$ topological $A$-modules and $\mathcal{X}$ a family of $A$-multilinear mappings from $E_{1} \times \cdots \times E_{n}$ into $F$. If the product of any neighborhood of 0 in $A$ by any neighborhood of 0 in $E_{i}$ is a neighborhood of 0 in $E_{i}(1 \leq i \leq n)$, then the equicontinuity of $\mathcal{X}$ at $(0, \cdots, 0)$ implies the equicontinuity of $\mathcal{X}$.

PROOF. Suppose that $\mathcal{X}$ is equicontinuous at $(0, \cdots, 0)$. Let $\left(a_{1}, \cdots, a_{n}\right) \in E_{1} \times \cdots \times E_{n}$ and let $W$ be a neighborhood of 0 in $F$. Define

$$
\mathcal{G}=\{H \subset\{1, \cdots, n\} ; H \neq\{1, \cdots, n\}\}
$$

There are neighborhoods $W_{H}$ of 0 in $F$ such that $\Sigma_{H \in \mathcal{G}} W_{H} \subset W$. For each $1 \leq i \leq n$ there is a neighborhood $U_{i}$ of 0 in $E_{i}$ so that the relations $z_{\imath} \in U_{i}$ imply

$$
v\left(z_{1}, \cdots, z_{n}\right) \in \bigcap_{H \in \mathcal{G}} W_{H} \text { for every } v \in \mathcal{X}
$$

Now, let $Z$ be a neighborhood of 0 in $A$ such that $Z a_{\imath} \subset U_{i}$ for every $1 \leq i \leq n$, and let $V_{i}$ be a neighborhood of 0 in $E_{\imath}$ such that

$$
V_{\imath} \subset U_{i} \cap Z U_{\imath} \cap Z^{2} U_{\imath} \cap \cdots \cap Z^{n-1} U_{i} \quad(1 \leq i \leq n) .
$$

Let $v \in \mathcal{X}$. We will prove that 


$$
v\left(a_{1}+x_{1}, \cdots, a_{n}+x_{n}\right)-v\left(a_{1}, \cdots, a_{n}\right) \in W
$$

for all $x_{\imath} \in V_{\imath}(1 \leq i \leq n)$. Indeed, for each given $H \in \mathcal{G}$, define

$$
v_{H}=v\left(y_{1}, \cdots, y_{n}\right)
$$

where $y_{z}=a_{\imath}$ if $i \in H$ and $y_{\imath}=x_{\imath}$ if $i \notin H$, take an $i_{0} \in\{1, \cdots, n\}-H$ and put $x_{\imath_{0}}=\lambda_{1} \times$ $\cdots \times \lambda_{\operatorname{Card}(H)} z_{\imath_{0}}$ (with $\lambda_{1}, \cdots, \lambda_{\operatorname{Card}(H)} \in Z$ and $z_{\imath_{0}} \in U_{\imath_{0}}$ ). Then

$$
v_{H}=v\left(z_{1}, \cdots, z_{n}\right)
$$

where $z_{\imath} \in U_{\imath}(1 \leq i \leq n)$. Therefore,

$$
v\left(a_{1}+x_{1}, \cdots, a_{n}+x_{n}\right)-v\left(a_{1}, \cdots, a_{n}\right)=\sum_{H \in \mathcal{G}} v_{H} \in \sum_{H \in \mathcal{G}} W_{H} \subset W
$$

This proves the theorem.

COROLLARY. Let $A$ be a commutative topological ring with identity, and let $n \in \mathbb{N}^{*}$. If $0 \in \overline{A^{\times}}$ (where $A^{\times}$denotes the multiplicative group of all invertible elements of $A$ ), then the following property is verified:

For all unitary topological $A$-modules $E_{1}, \cdots, E_{n}, F$ and all family $\mathcal{X}$ of $A$-multilinear mappings from $E_{1} \times \cdots \times E_{n}$ into $F$, the equicontinuity of $\mathcal{X}$ at $(0, \cdots, 0)$ implies the equicontinuity of $\mathcal{X}$.

REMARK 1. By the theorem, the conclusion of the corollary remains valid if we assume that $A$ is a commutative topological ring with the following property:

For every topological $A$-module $E$, the product of any neighborhood of 0 in $A$ by any neighborhood of 0 in $E$ is a neighborhood of 0 in $E$.

Nevertheless, if $A$ has identity, this is not a generalization. Indeed, suppose that there is a neighborhood $V$ of 0 in $A$ which has no invertible element. Let $E$ be the $A$-module $A$ endowed with its trivial topology (that is, the topology whose only open sets are $\emptyset$ and $E$ ). Then $E$ is a topological $A$ module and the product of $V$ by $E$ is not a neighborhood of 0 in $E$.

Let us now give an example which shows that the conclusion of the corollary is not always true (even for non-discrete topological rings) if $n \geq 2$.

EXAMPLE. Assume $n \geq 2$. Let $\left(A_{\imath}\right)_{\imath \in I}$ be a family of commutative rings with identity such that there is an $i_{0} \in I$ for which $A_{i_{0}}$ is not the zero ring. Consider each $A_{\imath}$ endowed with the discrete topology, and let $A$ be the product topological ring $\Pi_{i \in I} A_{i}$. We shall show that the conclusion of the corollary is false for the topological ring $A$. In order to do so, let $M$ be the product group $\left(A_{i_{0}}\right)^{N}$ endowed with the following law:

$$
\left(\left(a_{\imath}\right)_{i \in I},\left(x_{k}\right)_{k \in \mathbf{N}}\right) \in A \times M \mapsto\left(a_{i_{0}} x_{k}\right)_{k \in \mathbf{N}} \in M
$$

It is easily verified that $M$ is a unitary $A$-module. Let $E$ (resp. $F$ ) be the $A$-module $M$ endowed with the product topology (resp. the discrete topology). A simple argument shows that $E$ and $F$ are topological $A$-modules. Now, define

$$
f:\left(\left(x_{k}^{(1)}\right), \cdots,\left(x_{k}^{(n)}\right)\right) \in E^{n} \mapsto\left(x_{0}^{(1)} x_{k}^{(2)} \cdots x_{k}^{(n)}\right) \in F .
$$

Clearly, $f$ is $A$-multilinear. If $V=\left\{\left(z_{k}\right) \in E ; z_{0}=0\right\}$, then 


$$
f\left(\left(x_{k}^{(1)}\right), \cdots,\left(x_{k}^{(n)}\right)\right)=0
$$

for any $\left(\left(x_{k}^{(1)}\right), \cdots,\left(x_{k}^{(n)}\right)\right)$ in the open neighborhood $V \times E^{n-1}$ of $(0, \cdots, 0)$ in $E^{n}$. In particular, $f$ is continuous at $(0, \cdots, 0)$. Nevertheless, $f$ is discontinuous. Indeed, put

$$
\left(x_{k}^{(1)}\right)=(e, 0,0,0, \cdots) \text { and }\left(x_{k}^{(2)}\right)=\cdots=\left(x_{k}^{(n)}\right)=(e, e, e, \cdots)
$$

where $e$ denotes the identity of $A_{2_{0}}$. Let $V_{j}(1 \leq j \leq n)$ be a neighborhood of $\left(x_{k}^{(j)}\right)$ in $E$. We know that $V_{n}$ contains a neighborhood of $\left(x_{k}^{(n)}\right)$ of the form

$$
V_{n}^{\prime}=\left\{\left(z_{k}\right) \in E ; z_{\jmath}=x_{\jmath}^{(n)} \text { for } 0 \leq j \leq r\right\}
$$

Now, if $\left(c_{k}\right)=\left(x_{0}^{(n)}, \cdots, x_{r}^{(n)}, 0,0,0, \cdots\right)$, then

$$
\left(\left(x_{k}^{(1)}\right), \cdots,\left(x_{k}^{(n-1)}\right),\left(c_{k}\right)\right) \in V_{1} \times \cdots \times V_{n-1} \times V_{n}^{\prime} \subset V_{1} \times \cdots \times V_{n}
$$

and

$$
f\left(\left(x_{k}^{(1)}\right), \cdots,\left(x_{k}^{(n-1)}\right),\left(c_{k}\right)\right)=(\underbrace{e, \cdots, e}_{r \text {-times }}, 0,0, \cdots) \neq f\left(\left(x_{k}^{(1)}\right), \cdots,\left(x_{k}^{(n)}\right)\right)
$$

This establishes the discontinuity of $f$.

REMARK 2. Let $E$ and $F$ be as in the above example, and let $i$ be the identity mapping from $E$ into $F$. It is easily seen that $i$ is open, its kernel is closed and its graph is closed; however, $i$ is discontinuous. This shows, in particular, that the Open Mapping and Closed Graph Theorems are not always true in the context of complete metrizable topological modules, although they are known to be valid for a quite wide class of complete metrizable topological modules ([2], corollary 12.18).

\section{REFERENCES}

1. BOURBAKI, N., Espaces vectoriels topologiques, Actual. Scient. Ind., Chap. I et II, $n^{\circ}$ 1189, Hermann, Paris, 1966.

2. WARNER, S., Topological Fields, North-Holland Mathematics Studies 157, North-Holland, 1989. 


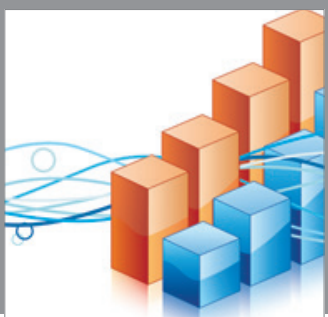

Advances in

Operations Research

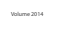

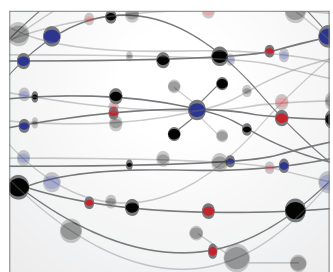

\section{The Scientific} World Journal
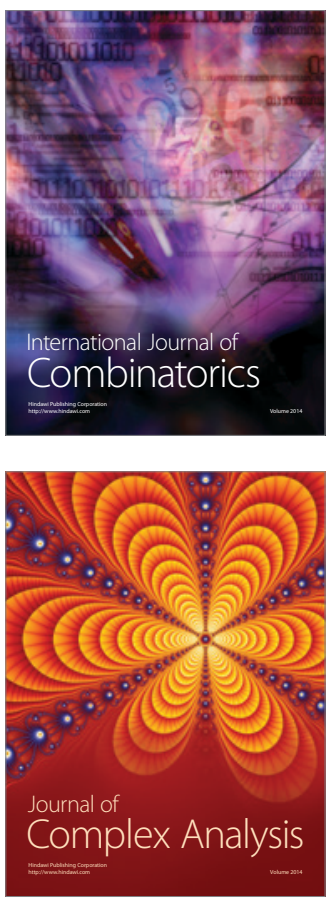

International Journal of

Mathematics and

Mathematical

Sciences
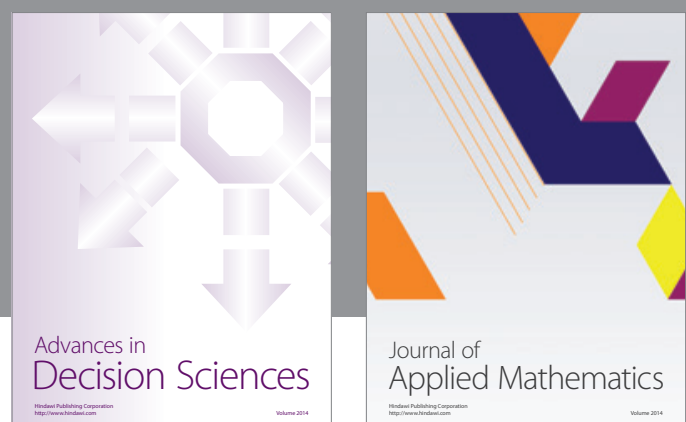

Journal of

Applied Mathematics
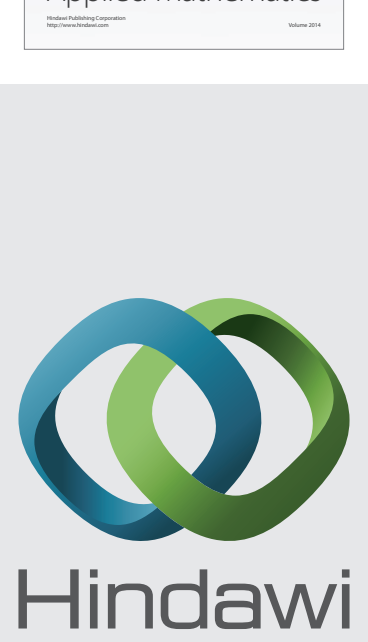

Submit your manuscripts at http://www.hindawi.com
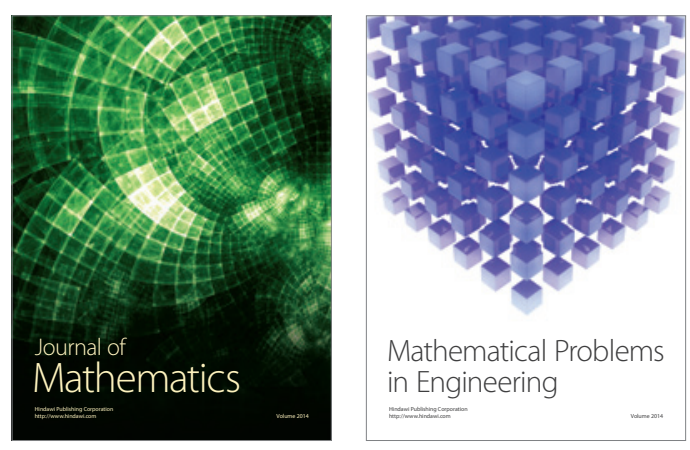

Mathematical Problems in Engineering
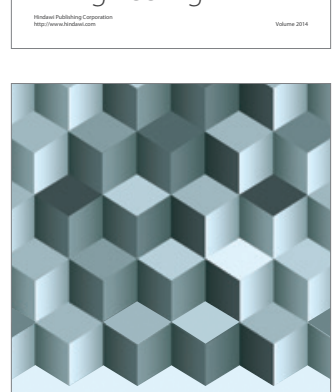

Journal of

Function Spaces
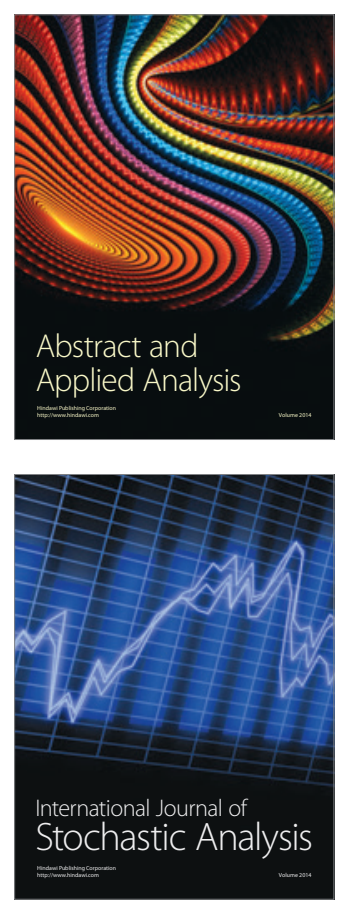

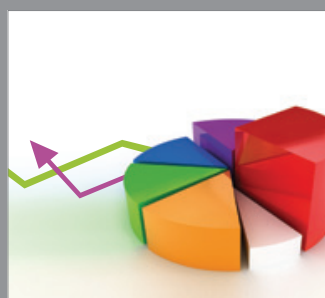

ournal of

Probability and Statistics

Promensencen
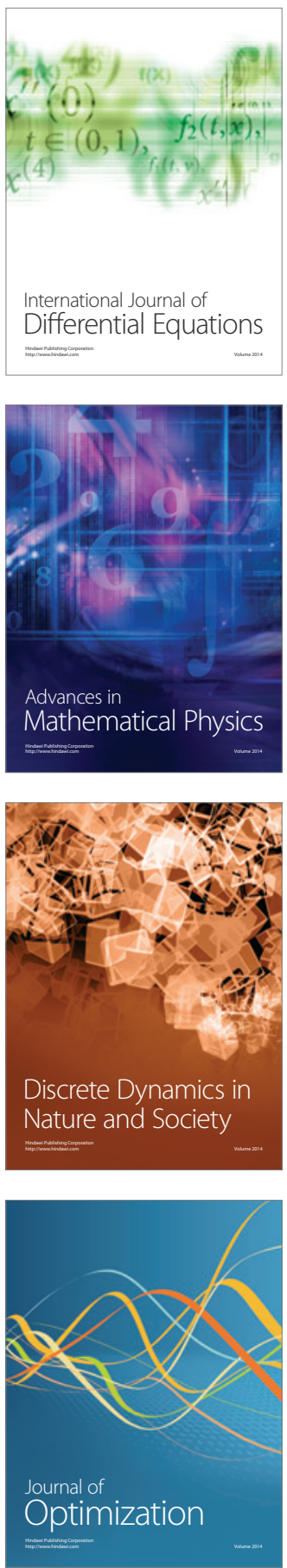